\title{
Overview of Malignant Solid Tumors in Neonates
}

\section{Karen S. Fernández ${ }^{1,2^{*}}$ and Reuben Antony ${ }^{3}$}

${ }^{1}$ Valley Children's Health Care, Cancer and Blood Disorders Center, 9300 Valley Children's Place, Madera, CA 93636, USA

${ }^{2}$ University of California San Francisco (UCSF)- Fresno, Pediatrics Hematolgy Oncology, 9300 Valley Children's Place, Madera, CA 93736, USA

${ }^{3}$ UC Davis Comprehensive Cancer Center, Pediatric Hematology and Oncology, UC Davis Children's Hospital, 2nd Floor, TICON 2 Building, 2516 Stockton Blvd, Sacramento, CA 95817, USA

\section{Abstract}

Solid tumors discovered at birth or during the first month after delivery are rare. Malignant tumors in Received: October 02, 2018 neonates represent only $2 \%$ of all malignancies in childhood. Some tumors which appear histologically Accepted: February 27, 2019 malignant may show benign behavior while apparently benign tumors may be fatal by virtue of their site Published: March 01,2019 of origin, which make neonatal tumors difficult diagnostic and therapeutic challenges in neonatal units. Planning diagnostic work-up and therapeutic interventions requires a multidisciplinary approach that involves, feto-maternal, neonatology, radiology, surgery, pathology, hematology, oncology and supportive care services. Here we provide a general overview of the most common malignant tumors seen in the newborn period.

\section{Publication History:}

\section{Keywords:}

Solid tumors, Cancer, Neoplasia, Newborns

\section{Introduction}

Neonatal tumors include a group of diverse neoplasms that are diagnosed prenatally or within the first 30 days of life. Only $2 \%$ of childhood tumors are reported to appear in the newborn period $[1,2]$. The incidence of malignant tumors is approximately 1 in 250,000 live births [2]. A majority of the tumors are histologically benign; however, they can be lethal due to their size and location. If malignant they are more likely to be the result of a genetic predisposition. The use of prenatal imaging has made antenatal diagnosis of solid tumors possible making their incidence appear higher than in the past decades.

The etiology of neonatal tumors while probably multifactorial is in most cases not known. Many neoplastic tumors that appear in utero are composed of embryonic or fetal tissues, suggesting a failure of proper maturation or unregulated cell growth during development. Tumor cells resemble undifferentiated cells seen during normal embryonic development, suggesting genetic changes which limit the cell's ability to fully differentiate as a possible mechanism [3].

Other possible causative factors in the development of tumors during the first month of life include: 1) genetic cancer predisposition 2) prenatal exposure to maternal cancer, chemotherapy and/ or radiotherapy, 3) prenatal exposure to toxins, 4) chromosomal abnormalities.

The frequency distribution of malignancies in the neonatal period is different from that found later in childhood (Figure 1).

The principles of managing malignant tumors in neonates are broadly the same as in older children, however, surgery plays the major part in the management of most neonatal benign and malignant tumors. A multidisciplinary team including neonatology, radiology, surgery, pathology, hematology, oncology, pharmacy and supportive care staff is necessary for optimal management for this reason, the management of this group of neonates should ideally be at a specialized center experienced in the medical and surgical care of neonates with cancer. In this review article we discuss the most common malignant tumors seen in the newborn period.

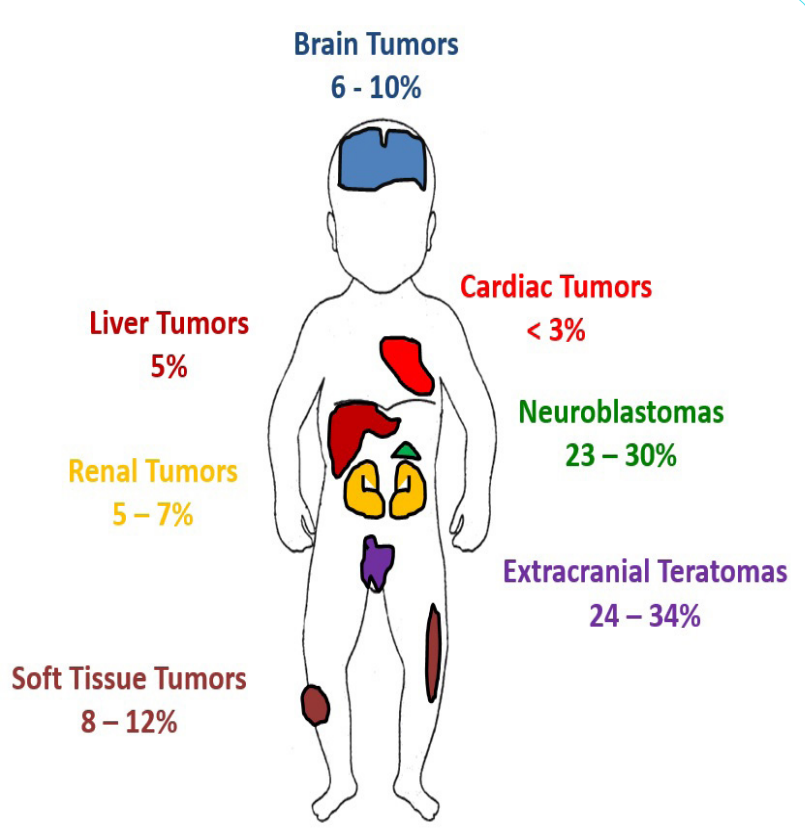

Figure 1: Frequency of Malignant Tumors in Neonates.

\section{Sacrococcigeal Teratomas}

Etiology and Incidence: Extracranial teratomas are the most common tumors in neonates, accounting for $28-39 \%$ of all tumors in the newborn period [4]. They occur in almost any midline location and are usually extragonadal. Teratomas contain tissue from two or three of the embryonic germinal layers: ectoderm, mesoderm, or

"Corresponding Author: Dr. Karen S. Fernández, Valley Children's Health Care, Cancer and Blood Disorders Center, 9300 Valley Children's Place, Madera, CA 93636, USA, E-mail: kfernandez@valleychildrens.org

Citation: Fernández KS, Antony R (2019) Overview of Malignant Solid Tumors in Neonates. Int J Pediatr Neonat Care 4: 147. doi: https://doi.org/10.15344/24552364/2019/147

Copyright: (C) 2019 Fernández et al. This is an open-access article distributed under the terms of the Creative Commons Attribution License, which permits unrestricted use, distribution, and reproduction in any medium, provided the original author and source are credited. 
endoderm. The most frequent sites are the sacrococcygeal or mediastinal areas. While usually isolated lesions, they may be part of the Currarino triad, which consists of a presacral tumor, anorectal malformation, and sacral anomaly. Sacrococcygeal teratoma (SCT) accounts for $35-60 \%$ of all teratomas seen in the postnatal period. They are more common in females with a predominance of $4: 1$.

Clinical features: SCT presents as a mass protruding between the coccyx and the rectum. The presentation is usually prenatal and they are easily distinguished from meningomyelocele or other tumors due to high tumor vascularity detected by ultrasound examination. With large tumors the affected fetus may develop anemia, high cardiac output failure, and ultimately hydrops. Teratomas can also appear in the neck or the mediastinum and present with polyhydramnios, because of ineffective fetal swallowing, or airway compression requiring in utero intervention or early delivery. If hydrops develops mortality is $100 \%$.

Diagnostics: Most teratomas are diagnosed during the routine 18 - 20-week prenatal scan gestation irrespective of the location of the lesion. Doppler ultrasound is the most useful diagnostic tool although fetal MRI provides better definition of complex lesions. If not already detected antenatally most SCTs are seen as a visible mass during neonatal newborn examination. Lesions with a large intrapelvic component may present with delayed symptoms of constipation, urinary retention and an abdominal mass. Thirty percent of SCT are mature but $10 \%$ may have yolk sac tumor elements, and the remainder have immature elements.

Treatment: Surgery remains the mainstay of treatment with complete excision of the tumor including the coccyx. Abdomino-perineal approach may be necessary for tumors with pelvic involvement. The incidence of malignancy increases with the age at diagnosis. The risk of malignancy is less than $10 \%$ at birth but more than $75 \%$ after 1 year. The risk of malignancy is high for incompletely excised tumors. The presence of malignant elements would necessitate usage adjuvant chemotherapy. Perinatal management of SCT includes consideration of Cesarean section delivery for tumors that are greater than $5 \mathrm{~cm}$ in diameter to avoid dystocia and tumor rupture.

\section{Neuroblastoma}

Etiology and Incidence: Neuroblastoma (NBL) is the most common malignant tumor found at birth. It occurs in the adrenal medulla or anywhere along the sympathetic neural chain along the cervical or thoracic areas. The clinical behavior of neuroblastoma in neonates ranges from spontaneous regression and resolution to aggressive malignant progression resistant to therapy. The cause of neuroblastoma is unknown. A familial tendency has been noted in $1-2 \%$, with mutations in anaplastic lymphoma kinase (ALK) gene; and mutations of the PHOX2B gene associated with other neural crest disorders like Hirschsprung's disease and central hypoventilation syndrome.

Clinical Features: In the newborn period, many infants are asymptomatic or may initially have only hepatomegaly (65\%) progressing to subcutaneous skin nodules (32\%). Other initial symptoms in newborns can be respiratory distress if the tumor is located in the posterior mediastinum or in the cervical region; Horner's syndrome is also a common presentation of cervical tumors and paraplegia or paralysis in case of intraspinal extension with spinal cord compression in the thoracic and lumbar spine [3]. Rarely acute visual loss could be the presenting symptom of metastatic neuroblastoma that compresses the optic nerve or the optic chiasm.

A palpable abdominal mass is the most common presentation in the neonatal period. The presence of bluish skin nodules described as "blueberry muffin spots" and liver enlargement are the most common indication of metastatic disease in neonates. However, these need careful differentiation from other causes of purpuric lesions that are more common in the neonatal period. Bone marrow involvement can cause cytopenias and bleeding symptoms. In contrast to older children, skeletal spread is rare in neonates.

Diagnostics: Widespread use of prenatal ultrasonography has led to increased prenatal detection. Neuroblastoma masses in cervical or thoracic locations can be detected antenatally. Differentiating a cystic neuroblastoma from adrenal hemorrhage by prenatal scan may be challenging and cause great parental anxiety. After birth, a radiograph, ultrasound, CT scan, or MRI and MIBG (Meta-IodoBenzyl-guanidine) scan can help with diagnosis and staging.

The finding of a solid suprarenal mass in association with liver lesions, makes the diagnosis of NBL highly likely. In this case testing for vanylmandelic acid (VMA) and homovanillic acid (HVA) in the urine is helpful. VMA and HVA are high in more than $90 \%$ of neonates with neuroblastoma. Random urine samples for catecholamine metabolites are sensitive and specific and have replaced 24-hour urine collections. Elevated VMA and HVA values together with bone marrow aspirates may be solely used for diagnosis where tissue diagnosis of the primary site is risky. The actual diagnosis of neuroblastoma is made by biopsy of the primary or metastatic tumor.

Neuroblastomas are staged according to the level of resectability of the primary site and the presence of metastasis. Various biomarkers, including N-MYC gene, histology and ploidy are taken into account to predict biologic behavior. Infants with neuroblastoma have a much better prognosis than older children mainly because their tumors are less likely to have amplification of the N-MYC gene, unfavorable histology or hypodiplody even in the presence of metastases to the liver, skin, and bone marrow. In recognition of the unique characteristics of neuroblastoma in the neonatal period, tumors in this age group have their own stage: $4 \mathrm{~S}$ disease (as long as bone marrow involvement is $<10 \%$ ), which generally carries a better prognosis. In cases in which the bone marrow has $>10 \%$ involvement the disease is classified as stage 4 .

Treatment: Treatment for neuroblastoma is determined by the stage of the disease, age of the child and biological features. Lesions identified prenatally as adrenal cysts require no specific treatment, but serial ultrasounds are recommended to help exclude differential diagnoses and detect complications. Cystic neuroblastoma with positive urinary catecholamines requires follow-up with monthly ultrasounds.

Asymptomatic, single solid masses presenting postnatally are generally managed with observation alone, with further intervention necessary only if the mass fails to resolve with time, or complications develop [4]. If obstruction or compression of vital organs occurs, surgical removal may be necessary. Infants with early-stage neuroblastoma without metastasis are usually treated with surgical resection alone. If the tumor cannot be completely removed, chemotherapy is recommended. The chemotherapy regimen depends on the stage and biological features and varies from 2 - 8 cycles. Radiation therapy is usually used for older children. Overall five-year survival rates are 
approximately 80 percent for infants diagnosed at younger than one year of age and 95 percent for infants with localized tumors [4].

\section{Soft Tissue Tumors}

Etiology and incidence: A majority of soft tissue tumors (STT) that occur in the newborn are pathologically benign (75\%). Among children diagnosed with STT $20 \%$ present in infants between birth and 12 months of age. The exact incidence in the first 4 weeks of life is not really known. The common neonatal benign STT are hemangioendothelioma, lymphangioma, and fibromatosismyofibromatosis while fibrosarcoma and rhabdomyosarcoma are malignant STT frequently seen in this age.

Clinical features: Head and neck regions are the most frequent sites of STT in newborns They develop in the extremities, more frequently after the first year of life. Occasionally some STT may arise from visceral organs.

Diagnostics: This tumor are discovered during prenatal ultrasound or immediately after birth when a mass is identified. The definitive diagnosis is made with biopsy, histopathology examination and molecular profiling.

Treatment: STT are treated primarily by wide surgical resection. The subsequent histopathologic diagnosis guides additional treatment with chemotherapy or targeted therapies.

Below we describe some of the STT commonly diagnosed in the newborn period and outline specific clinical findings and treatment.

Fibromatoses are fibroblastic proliferations of spindle cells which are locally invasive, have a high potential for recurrence, but do not metastasize. They include infantile fibromatosis, fibromatosis colli, and infantile digital fibromatosis.

Infantile fibromatosis, also known as myofibromatosis or hemangiopericytoma, is the most common fibrous tumor of infancy. It occurs in three forms: solitary, multicentric, or generalized. Solitary is the most common form. These tumors often occur in the head and neck region and have very good outcomes, seldom recur and do not metastasize. Multicentric myofibromatosis can have involvement of skin, soft tissue, and less often bone. It usually has a good outcome and can spontaneously regress [7]. However, increased morbidity is seen with vertebral bone lesions due to pathologic fractures and risk of spinal cord compression [8]. Generalized myofibromatosis is associated with visceral involvement and is often fatal. Generalized myofibromatosis is associated with mutations in the PDGFRB gene which may be sensitive to tyrosine kinase inhibition with imatinib or desatinib [9].

Infantile desmoid-type fibromatosis is analogous to desmoid tumors in older children, which are rare, locally invasive tumors that occur in deep soft tissues. Surgical excision is the treatment of choice, but these tumors tend to recur without metastatic spread.

Fibromatosis coli occurs as a mobile neck mass between the second and fourth weeks after birth. It may be associated with birth trauma and spontaneously resolves.

Sarcomas in infants are rare, accounting for approximately $10 \%$ of neonatal cancers. The most common sarcoma in the newborn period are fibrosarcomas, and rhabdomyosarcomas [7]. They arise primarily from connective tissues such as fibrous tissue, adipose tissue, and muscle tissue in the head and neck or the genitourinary region. In general, males and children of African descent have a slightly higher incidence than females and Caucasian children. They are also found in association with various syndromes including Li-Fraumeni syndrome, and neurofibromatosis. Sarcomas need surgical resection and depending on the surgical staging, chemotherapy may need to be considered carefully in this particular vulnerable period of life.

Infantile fibrosarcomas (IFS) are sarcomas that commonly show up in the extremities. IFS is a distinct entity from fibrosarcoma which occurs later in childhood or adulthood. Almost all IFS have a characteristic chromosomal translocation, $t$ (12:15), involving the ETV6 and NTRK3 genes [8]. Metastatic disease is rare, but CT of the chest, abdomen and pelvis and bone scan are indicated for a complete evaluation. Complete surgical excision, is usually curative. Recurrent fibrosarcoma may require second local excision and chemotherapy. Occasionally, amputation may be necessary for extremity lesions. For patients with unresectable tumors chemotherapy is recommended. More recently the introduction of NTRK inhibitors such as Larotrectinib for patient that harbor a pathogenic NTRK gene fusion has increased our ability to achieve tumor control medically and sometimes avoid mutilating surgery. Overall survival for infants with infantile fibrosarcoma is close to 100 percent.

Rhabdomyosarcoma (RMS) is the most common soft tissue sarcoma in children but constitutes only $2 \%$ of the cases presenting in the newborn period. They usually present as an enlarging mass with associated symptoms based on mass effect on nearby structures. Fifty percent of the RMS in neonates appear in the sacrococcygeal, perirectal, bladder and vagina areas, but they can also be seen in the thorax abdomen, pelvis, and extremities. The two major histologic subtypes of RMS are embryonal and alveolar - these have different clinical behaviors. Embryonal rhabdomyosarcomas are most common in neonates, they are associated with allelic loss of the $11 \mathrm{p} 15$ regions. Alveolar rhabdomyosarcomas are associated with either $t(2 ; 13)$ or $t$ $(1 ; 13)$ a feature associated with poor prognosis [10]. The treatment of choice for RMS is a combination of wide surgical excision and either pre or postoperative chemotherapy. Surgery alone or chemotherapy alone carries high recurrence rate. Good prognostic sites in RMS are the genitourinary and the head and neck lesions, whereas the trunk and extremities carry a poor prognosis.

\section{Brain Tumors}

Etiology and incidence: Neonatal brain tumors comprise $<1 \%$ of all pediatric brain tumors [12]. In neonates they are more often supratentorial than in older children. Teratomas and astrocytoma are relatively common, with medulloblastoma, choroid plexus carcinoma, peripheral neuro-ectoderm tumors (PNET), meningioma, craniopharyngioma and ependymoma occurring less frequently. When an infratentorial tumor is present in a newborn a consideration is the presence of an atypical teratoid rhabdoid tumor (AT/RT) of the brain, which is likely to be associated with INI1 gene deletion.

Within the subgroup of perinatal/neonatal astrocytomas high grade gliomas occur more frequently than low grade astrocytomas in contrast to older children in whom low grade astrocytomas are significantly more frequent [13].

Germ cell tumors of the brain tend to be large tumors that can displace a significant portion of the brain which may cause perinatal death. 
Citation: Fernández KS, Antony R (2019) Overview of Malignant Solid Tumors in Neonates. Int J Pediatr Neonat Care 4: 147. doi: https://doi.org/10.15344/24552364/2019/147

Page 4 of 7

\begin{tabular}{|c|c|c|c|}
\hline Tumor & Incidence in Neonates & Clinical Findings & $\begin{array}{l}\text { Genetic Alterations and Associated } \\
\text { Syndromes and Conditions }\end{array}$ \\
\hline $\begin{array}{l}\text { Sacrococcygeal } \\
\text { Teratomas }\end{array}$ & $35-60 \%$ of all teratomas & $\begin{array}{c}\text { Hydrops fetalis } \\
\text { Large sacral mass } \\
\text { Anorectal malformation }\end{array}$ & $\begin{array}{c}\text { Currarino Triad } \\
\text { Klinefelter Syndrome }\end{array}$ \\
\hline Neuroblastoma & $20 \%$ of all NBL & $\begin{array}{l}\text { Hepatomegaly } \\
\text { Skin nodules } \\
\text { Heterochromia iridis } \\
\text { Horner Syndrome }\end{array}$ & $\begin{array}{l}\text { ALK gene mutations } \\
\text { PHOX2B gene mutations } \\
\text { Hypoventilation syndrome } \\
\text { Hirschsprung's disease }\end{array}$ \\
\hline $\begin{array}{l}\text { Soft Tissue Tumors, } \\
\text { Sarcomas }\end{array}$ & $10-15 \%$ of all STT & $\begin{array}{l}\text { Abdominal mass } \\
\text { Hematuria } \\
\text { Soft Tissue mass }\end{array}$ & Li-Fraumeni \\
\hline Disseminated Fibromatosis & $?$ & Visceral Tumors & PDGFRB gene mutations \\
\hline Infantile Fibrosarcoma & $?$ & $\begin{array}{l}\text { Soft tissue tumors in the } \\
\text { extremities }\end{array}$ & ETV6 and NTRK3 gene mutations \\
\hline ATRT of the Brain & Very Rare? & $\begin{array}{c}\text { Brain mass } \\
\text { Hydrocephalus }\end{array}$ & INI1 mutation \\
\hline $\begin{array}{l}\text { Subependymal Giant Cell Astrocytoma } \\
\text { (SEGA) }\end{array}$ & $2.2 \%$ are congenital & $\begin{array}{c}\text { Brain mass } \\
\text { Hydrocephalus }\end{array}$ & Tuberous Sclerosis \\
\hline Hepatoblastoma & $10 \%$ of all HBL & Abdominal Mass & $\begin{array}{c}\text { Beckwith-Wiedemann } \\
\text { Li-Fraumeni } \\
\text { FAP } \\
\text { Trisomy } 18 \\
\end{array}$ \\
\hline Wilms Tumor & $0.2 \%$ of all WT & $\begin{array}{l}\text { Abdominal mass } \\
\text { Hypertension } \\
\text { Hematuria }\end{array}$ & $\begin{array}{c}\text { Beckwith-Wiedemann } \\
\text { WAGR } \\
\text { Denys-Drash } \\
\text { Simpson-Golabi-Behmel } \\
\text { Hemihypertrophy } \\
\text { Soto's Syndrome }\end{array}$ \\
\hline $\begin{array}{l}\text { Malignant Rhabdoid Tumor of the Kidney } \\
\text { (MRTK) }\end{array}$ & & $\begin{array}{l}\text { Abdominal mass } \\
\text { Hypertension } \\
\text { Hematuria }\end{array}$ & INI1 mutation \\
\hline
\end{tabular}

Table 1. Tumors in Neonates and Associated Genetic Abnormalities and Syndromes

Congenital subependymal giant cell astrocytoma (cSEGA), a low-grade astrocytoma that arises within the ventricles of the brain can also be seen in the newborn period, and it is most commonly associated with tuberous sclerosis complex. Although a low-grade tumor, its location can potentially obstruct the ventricles and lead to hydrocephalus.

Clinical Features: Brain tumors in neonates can present at different time points: prenatal period, perinatal and within the first month after birth [14]. Differential diagnoses of neonatal CNS tumors include cerebral infarction, intracranial hemorrhage or vascular malformations.

Clinical consequences of perinatal/neonatal brain tumors are premature labor, cephalo-pelvic disproportion, still birth, hydrocephalus, bulging fontanelles, seizures, focal neurological abnormalities such as hemiparesis, cranial nerve palsies, vomiting, apnea, failure to thrive and intracranial hemorrhage [15]. The diagnosis may be delayed due to the expansile nature of the neonatal skull which silently accommodates the growing tumor, non-specific clinical symptoms and low level of suspicion.

Diagnostics: In the prenatal period detection of increased fetal head circumference, hydrocephalus or an intracranial mass by antenatal ultrasound should prompt more detailed analysis by either ultrasound or by fetal MRI [14]. After birth brain and spinal cord
MRI is the imaging modality of choice. Brain tumors which usually remain localized in older pediatric patients can be disseminated at presentation in neonates. Radiological features such as location, solid/ cystic structure, pattern of contrast enhancement, calcification and diffusion restriction can be used to make a radiological diagnosis. If germ cell tumor is included in the radiological differential diagnosis then serum and cerebrospinal fluid alpha-fetoprotein (AFP) and beta human chorionic gonadotropin ( $\beta-\mathrm{HCG})$ need to be evaluated.

Treatment: During the neonatal period the treatment of brain tumors is challenging due to their rarity, and limited therapeutic options, increased surgical complications, perioperative mortality and aggressive biological behavior. Treatment consists of either surgical resection and/or chemotherapy.

Surgical resection is the primary treatment. Surgery is performed for CSF diversion to relieve hydrocephalus or to obtain tissue for analysis and for tumor resection. While a safe complete resection should be considered in all cases this is often not possible due to the large size of the tumor (which can sometimes occupy a large part of the cranium) and increased vascularity of the tumor.

Neoadjuvant therapy using chemotherapy has been used to reduce tumor size and vascularity and to facilitate gross total tumor resection and minimize intraoperative blood loss [16, 17]. For specific pathologies such as cSEGA the use of targeted therapies 
such as Everolimus an m-TOR inhibitor has shown to efficacious in decreasing the size of this tumors.

Radiation therapy is contraindicated in this age group due to the severe long terms' effects on growth, cognition and neuro-endocrine function.

The prognosis of neonates with CNS tumors is determined by tumor location, size, dissemination, histology and potential for resectability. The prognosis is better for infants who have benign extra-parenchymal tumors (e.g. Choroid Plexus Papilloma) compared to infants who have deep seated malignant tumors which tend to invade surrounding structures (e.g. PNET) [18]. Isaac reported an overall survival of $46 \%$ with survival significantly higher in neonates with benign tumors [12]. Among the children who become long term survivors there is a disproportionately high incidence of neurological, cognitive and endocrine deficits.

\section{Renal Tumors}

Etiology and incidence: The majority of renal masses that occur in the neonate are malformations, such as hydronephrosis and polycystic kidney disease. The most common tumor of the kidney in the neonate is congenital mesoblastic nephroma (CMN), accounting for approximately $75 \%$ of the renal neoplasms. It is a tumor almost exclusively found during the first 6 months of life and it is usually unilateral. Histologically, it is a mesenchymal tumor composed predominantly of fibroblastic cells. Two variants have been identified: classical and cellular. Although, the natural history of CMN is benign, recurrence and metastases have been described. Cellular CMN appears to be characterized by a specific chromosomal translocation $t$ $(12 ; 15)$ which has been shown to directly confer oncogenic potential [19].

Wilms Tumor (WT), also known as nephroblastoma is unusual in neonates. Although it is the most common abdominal tumor in children, only $0.2 \%$ occur during the neonatal period. WT is associated in $25 \%$ of the cases with several recognizable syndromes including: WAGR (Wilms, aniridia, genitourinary tract abnormalities, mental retardation), Denys-Drash associations and overgrowth syndromes such as Beckwith-Weidemann syndrome (gigantism, omphalocele, macroglossia, hemihypertrophy) and other overgrowth syndromes such as Simpson-Golabi-Behmel syndrome or Soto's syndrome [20, 21]. Two chromosomal loci have been implicated in the pathogenesis of Wilms Tumor: WT1 11p13 and WT2 11p15, however most cases of WT do not have mutations in these genes.

Other renal neoplasms seen in the newborn period are malignant rhabdoid tumor of the kidney (MRTK) and clear cell sarcoma of the kidney. MRTK tend to metastasize and present with advanced stage at diagnosis. MRTK can occur extrarenally, particularly in the brain, and therefore the finding of MRTK warrants brain MRI. MRTK tends to be very aggressive with $>90 \%$ mortality. Most rhabdoid tumors whether they occur in the, kidney or elsewhere, have INI1 deletions. INI1 is a tumor suppressor gene also known as SMARCB1 and hSNF5 that predisposes children to malignancies due to overexpression of EZH2 gene. Identification of INI1 facilitates accurate diagnosis of rhabdoid tumors. Patients found to have this mutation need to have genetic counseling regarding its inheritance.

Clear cell sarcoma of the kidney is extremely rare in neonates, but unlike the poor prognosis seen in older children, neonates seem to have a better outcome.
Clinical features: Polyhydramnios or the finding of an abdominal mass during routine antenatal ultrasound is the most common presentation of renal tumors. Diagnosis in the neonatal period remains more common than antenatal diagnosis with around $75 \%$ of perinatal cases of CMN diagnosed after birth. The most common finding is an asymptomatic abdominal mass although hematuria, hypertension, and hypercalcemia have all been reported and do not appear to be specific for tumor type.

Diagnostics: Ultrasound remains the first line investigation, but has been reported to underestimate the local and regional extent of the mass. MRI remains the gold-standard imaging modality in order to guide management and prognostication, but it is limited in distinguishing between CMN and WT. Because WT metastasizes to the lungs a chest CT must also be included in the evaluation.

Treatment: The majority of $\mathrm{CMN}$ are confined to the renal capsule, and therefore usually cured with surgical excision (nephrectomy) alone. Most WT occurring in the neonatal period are usually completely resected and classified as stage I and II. In infants with bilateral WT the approach to resection is more conservative and chemotherapy is preferred over surgery as a nephron sparing approach. The treatment of MRTK also requires surgery and chemotherapy. The recent introduction of EZH2 inhibitors such as Tazemetostat a inhibitor of EZH2 could benefit patients with MRTK.

\section{Hepatoblastoma}

Etiology and incidence: Hepatoblastoma (HBL) is the most common primary liver malignancy in neonates, but only $10 \%$ of all hepatoblastomas occur in the first 30 days after birth. It has been associated with genetic syndromes, including Beckwith-Wiedemann syndrome, Li-Fraumeni syndrome, familial adenomatous polyposis, and trisomy 18 and other chromosomal abnormalities [22]. Several recent studies have shown that the incidence of HBL in the neonatal period has been increasing over the past 20 years, which may reflect the improved survival of preterm infants. These findings suggest that some aspects of treatment for premature infants maybe carcinogenic or that the etiology of HBL is linked to low birth weight.

Clinical features: The most common initial finding for liver tumors is a mass detected on routine ultrasound scan requested due to abdominal distension. Some liver tumors are detected in utero as early as 16 weeks gestation [22].

Diagnostics: HBL usually presents as a well- defined, hyper-echoic, solid and non-cystic intra-hepatic mass, commonly located in the right lobe of the liver in $\sim 70 \%$ of the cases. Lungs are the most common site of metastatic disease. CT scan of the chest and CT or MRI of the abdomen are indicated for staging purposes. Alpha fetoprotein is markedly elevated usually in the range of several thousands in $90 \%$ of $\mathrm{HBL}$, and when low $(<100 \mathrm{pg} / \mathrm{dL})$ confers poor prognosis.

Treatment: Complete resection is the most important factor for cure. Resection followed by chemotherapy is the treatment of choice. Chemotherapy is given pre-operatively in order to allow complete surgical resection. Congenital HBL does not confer worse outcome than those discovered at an older age [23]. The treatment approach for newborns with HBL should be similar to that for older children, provided that they are stable enough to undergo proper staging, surgical resection and chemotherapy. 


\section{Special Considerations for Neonates with Cancer}

\section{Diagnostics}

The diagnosis and treatment of neonatal solid tumors, benign or malignant, is difficult and complicated. The ability to diagnose neoplasms in utero has evolved significantly with improvement in imaging technology. Fetal ultrasonography, magnetic resonance imaging (MRI), and echocardiography offer physicians the opportunity to diagnose a variety of congenital diseases in the prenatal period $[24,25]$.

Ultrasonography is a critical component of the prenatal obstetric evaluation and is currently the standard of care [25]. Ultrasound and MRI are preferred because they avoid the damaging effects of ionizing radiation.

For decades, ultrasonography has been the principal imaging modality for prenatal diagnosis of fetal anomalies. Ultrasound is widely available, non-expensive, noninvasive and provides real-time evaluation of the fetus. However, one shortcoming of ultrasound is that results are operator dependent and can vary with the experience and expertise of the examiner [26]. Over the past 20 years, magnetic resonance imaging (MRI) has become increasingly utilized as an adjunct to ultrasound in the evaluation of fetal and neonatal anomalies. Ultrafast MRI, which limits fetal motion artifact and does not require maternal-fetal sedation is now available and frequently utilized [26, 27].

The greatest utility of MRI appears to occur in cases where sonographic findings are equivocal or further diagnostic information is desired or a better understanding of the anatomic relationships between neoplasms and adjacent structures [28].

The toxicity of gadolinium in the growing fetus has to be considered when deciding on the use of MRI. Gadolinium contrast dye to enhance fetal imaging is administered as a gadolinium-chelate molecule that is water-soluble and can cross the placenta into fetal circulation. Following filtration and excretion by the fetal kidneys, these molecules can accumulate in amniotic fluid with the ability to release potentially toxic gadolinium ions [29]. Although the effect of free gadolinium ions on the fetus is unknown, the current recommendation is to abstain from the routine use of contrast MRI unless the benefits can be shown to clearly outweigh this theoretical risk.

On the other hand, the American College of Obstetricians and Gynecologists states in its most recent Guidelines for Diagnostic Imaging in Pregnancy and Lactation that there is no evidence of harm from tissue heating, acoustic damage, or teratogenesis when using MRI in pregnancy [30].

\section{Delivery}

Having diagnostic imaging that points to anomalies allows the care team to adequately plan a safe delivery (vaginal vs Cesarean section) of the neonate as well as select an appropriate center for delivery. Most fetuses, diagnosed prenatally with a tumor are delivered in a tertiary center were a comprehensive multidisciplinary team is available. The personnel required for the birth aside from those needed to care for the mother: neonatologist, pediatric anesthesiologist, pediatric otolaryngologist, pediatric general surgeon, pediatric cardiothoracic surgeon need to prepare and coordinate the delivery, diagnostic procedures and neonatal operative services. Early diagnosis also offers the possibility of using the ex utero intrapartum treatment (EXIT) approach particularly for cervical teratomas [31, 32]. In the EXIT procedure, the fetus is partially delivered by cesarean section and undergoes surgical procedures while the placenta and the umbilical cord remain intact.

\section{Chemotherapy and radiation}

The principles of managing malignant disease are the same as in older children. However, surgery plays the major part in the management of most benign and many malignant tumors. Neonates, due to their immaturity and physiology are at increased risk of sideeffects from chemotherapy and radiation. The role of chemotherapy must be carefully considered on an individual basis. Chemotherapy doses should be calculated according to body weight rather than body surface area. Special attention to the many factors that affect drug pharmacokinetics in the newborn period is needed. Intrinsic neonatal factors that may result in a newborn having a suboptimal amount of drug for effective cancer treatment or prolonged exposure leading to increased toxicities are: 1) higher total body water 2) lower amount of adipose tissue than older children, 3)altered fetal drug binding by albumin 4) reduced neonatal kidney function at $20-40 \%$ of adults 5) immaturity of neonatal liver effecting its ability metabolize and excrete drugs, 6) metabolic enzymes concentrations are much lower in neonates. Radiation therapy is avoided in neonates as it disrupts the growth and development of normal tissues.

\section{Targeted therapies}

As the therapeutic landscape for pediatric cancer evolves, the discovery and introduction of novel therapeutic molecules targeting specific cancer driving pathways in increasing.

While the rationale to use targeted therapies especially when the target and related pathway have been well characterized may be compelling $\mathrm{t}$ is important to use these new agents in the context of clinical trials so that data regarding efficacy and toxicity can be collected and disseminated.

\section{Psychosocial aspects}

The diagnosis of a neonatal tumor has a profound emotional impact on a family and raises questions about accuracy of diagnosis, prognosis, treatment options, and long-term outcomes [33]. While technological improvements in imaging modalities have led to better sensitivity in diagnosing congenital anomalies, the false-positive rate needs to also be considered while taking clinical decisions [34].

Careful considerations should be taken in while interpreting prenatal imaging as normal anatomic variants can have the appearance of a neoplastic process and some lesions will regress spontaneously. Conversely, sonographic findings of polyhydramnios, fetal hydrops, hepatosplenomegaly, and placentomegaly should raise suspicion for an undiagnosed tumor.

Psychosocial and spiritual support should be offered to the parents facing the birth of an infant with tumor, or to those whose tumor is diagnosed after birth. Anticipating that the infant may experience loss of physical, physiological and/or cognitive function, families should be supported with psychological, pastoral and grieving services. 
Citation: Fernández KS, Antony R (2019) Overview of Malignant Solid Tumors in Neonates. Int J Pediatr Neonat Care 4: 147. doi: https://doi.org/10.15344/24552364/2019/147

\section{Conclusions}

Tumors during the first month after birth are extremely rare. The presence of a malignant tumor in the newborn period should rise the possibility of a tumor predisposition syndrome and relevant screen should be performed looking for germline or somatic mutations. Advances in prenatal diagnostic imaging allows early detection of solid tumors. The diagnosis and treatment of newborns with malignant solid tumors is complex due to the unique physiology of the neonate. In expert hands, neonatal tumors generally carry the same or better prognosis than the same tumors in older children. Neonatal tumors should be treated as far as possible in specialized centers with a high level of multidisciplinary expertise.

\section{Competing Interests}

The author declare that no competing interests is present.

\section{References}

1. Moore SW, Satge D, Sasco AJ, Zimmermann A, Plaschkes J (2003) The epidemiology of neonatal tumours. Report of an international working group. Pediatr Surg Int 19: 509-519.

2. Bader JL, Miller RW (1979) US cancer incidence and mortality in the first year of life. Am J dis Child 133: 157-159.

3. Lakhoo K, Sowerbutts H (2010) Neonatal tumours. Pediatr Surg Int 26 1159-1168.

4. Campbell AN, Chan HSL, O'Brien A, Smith CR, Becker LE (1989) Malignancy in the neonate. Med Pediatr Onc 17: 101

5. Nuchtern JG (2006) Perinatal neuroblastoma. Semin Pediatr Surg 15: 10-16

6. Cozzi DA, Mele E, Ceccanti S, Natale F, Clerico A, et al. (2013) Long-term follow-up of the "wait and see" approach to localized perinatal adrenal neuroblastoma. World J Surg 37: 459-465.

7. Gopal M, Chahal G, Al-Rifai Z, Eradi B, Ninan G, et al. (2008) Infantile myofibromatosis. Pediatr Surg Int 24: 287-291.

8. Buonuomo PS, Ruggiero A, Zampino G, Maurizi P, Attina G, et al. (2006) A newborn with multiple fractures as first presenation of infantile myofibromatosis. J Perinatol 26: 653-655.

9. Arts FA, Sciot R, Brichard B, Renard M, de Rocca Serra A, et al. () PDGFRB gain-of-function mutations in sporadic infantile myofibromatosis. Hum Mol Genet 26: 1801-1810.

10. Lobe TE, Wiener ES, Hays DM, Lawrence WH, Andrassy RJ, et al. (1994) Neonatal rhabdomyosarcoma: the IRS experience. J Pediatr Surg 29: 1167 1170 .

11. Russell H, Hicks MJ, Bertuch AA, Chintagumpala M (2009) Infantile fibrosarcoma: clinical and histologic responses to cytotoxic chemotherapy. Pediatr Blood Cancer 53: 23-27.

12. Rickert CH (1999) Neuropathology and prognosis of foetal brain tumors. Acta Neuropathol 98: 567-576.

13. Isaacs H Jr (2016) Perinatal (fetal and neonatal) astrocytoma: a review. Childs Nerv Syst 32: 2085-2209.

14. Bodeliwala S, Kumar V, Singh D (2017) Neonatal Brain Tumors: A Review. J Neonatal Surg 6: 30.

15. Volpe JJ, Robinson S (1995) Chapter 37-Brain tumors and vein malformations In: Volpe JJ, editor. Neurology of the newborn, (3rd ed) Philadelphia: WB Saunders, pp. 795-807.

16. Iwama J1, Ogiwara H, Kiyotani C, Terashima K, Matsuoka K, et al. ()2015 Neoadjuvant chemotherapy for brain tumors in infants and young children. J Neurosurg Pediatr 15: 488-492.

17. Van Poppel M, Klimo P Jr, Dewire M, Sanford RA, Boop F, et al. (2011) Resection of infantile brain tumors after neoadjuvant chemotherapy: The St. Jude experience. J Neurosurg Pediatr 8: 251-256.
18. Sugimoto $M$, Kurishima C, Masutani S, Tamura M, Senzaki H et al. (2015) Congenital Brain Tumor in the first 2 months of life. Pediatr Neonatol 56: 369-375.

19. Isaacs H Jr (2008) Fetal and neonatal renal tumors. J Pediatr Surg 43: $1587-$ 1595.

20. Lakhoo K, Sowerbutts H (2013) Neonatal tumours. Pediatr Surg Int 26:11591168.

21. Lapunzina P (2005) Risk of tumorigenesis in overgrowth syndromes: a comprehensive review. Am J Med Genet C Semin Med Genet 137C: 53-71.

22. Gracia Bouthelier R, Lapunzina $P$ (2005) Follow-up and risk of tumors in overgrowth syndromes. J Pediatr Endocrinol Metab 1: 1227-1235.

23. Isaacs H Jr (2007) Fetal and neonatal hepatic tumors. J Pediatr Surg 42 1797-1803.

24. Trobaugh-Lotrario AD, Chaiyachati BH, Meyers RL, Häberle B, Tomlinson $\mathrm{GE}$, et al. (2013) Outcomes for patients with congenital hepatoblastoma. Pediatr Blood Cancer 1817-1825.

25. Santos XM, Papanna R, Johnson A, Cass DL, Olutoye OO, et al. (2010) The use of combined ultrasound and magnetic resonance imaging in the detection of fetal anomalies. Prenat Diagn 30: 402-407.

26. Lee TC, Olutoye OO (2012) Evaluation of the prenatally diagnosed mass. Semin Fetal Neonatal Med 17: 185-191.

27. Chung R, Kasprian G, Brugger PC, Prayer D (2009) The current state and future of fetal imaging. Clin Perinatol 36: 685-699.

28. Pugash D, Brugger PC, Bettelheim D, Prayer D (2008) Prenatal ultrasound and fetal MRI: The comparative value of each modality in prenatal diagnosis. Euro J Radiol 68: 214-226.

29. Breysem L, Bosmans H, Dymarkowski S, Schoubroeck DV, Witters I, et al. (2003) The value of fast MR imaging as an adjunct to ultrasound in prenatal diagnosis. Euro Radiol 13: 1538-1548.

30. Expert Panel on MR Safety, Kanal E, Barkovich AJ, Bell C, Borgstede JP, et al. (2013) ACR guidance document on MR safe practices: 2013. Expert Panel on MR Safety. J Magn Reson Imaging 37: 501-530.

31. The American College of Obstetricians and Gynecologists (2016) Committee Opinion No. 656: Guidelines for Diagnostic Imaging During Pregnancy and Lactation. Obstetrics \& Gynecology 127: e75-e80.

32. Albanese CT (2005) The EXIT strategy. Neo Reviews 6: e431-e435.

33. Bouchard S, Johnson MP, Flake AW, Howell L, Myers LB, et al. (2002) The EXIT procedure: experience and outcome in 31 cases. J Pediatr Surg 37: 418-426.

34. Borsellino A, Zaccara A, Nahom A, Trucchi A, Aite L, et al. (2006) Falsepositive rate in prenatal diagnosis of surgical anomalies. J Pediatr Surg 41: 826-829.

35. Isaacs H Jr (1987) Congenital and neonatal malignant tumors. A 28-year experience at Children's Hospital of Los Angeles. Am J Pediatr Hematol Oncol 9:121-129.

36. Kuki I, Kawawaki H, Okazaki S, Ehara E, Yoshida Y, et al. (2018) Efficacy and safety of everolimus in patient younger than 12 months with congenital subependymal giant cell astrocytoma. Brain \& Development 40: 415-420. 